\title{
Vacunación para odontólogos. Encuentros cercanos de primer contacto.
}

\author{
Vaccination for dentists. \\ Close encounters of first contact.
}

\begin{abstract}
L os odontólogos, por lo general, no estamos en un hospital como primera línea de atención COVID, pero sí somos el PRIMER CONTACTO de pacientes asintomáticos con SARS-CoV-2. Ante la falta de apoyo al personal de salud privado, dedicamos esta editorial para reforzar los argumentos para solicitar la vacunación a todos. Tenemos encuentros cercanos de primer contacto.

Debe quedar bien claro que los profesionales de la odontología somos un grupo de ALTO RIESGO de infección y GRUPO VULNERABLE, ya que prestamos SERVICIOS DE SALUD en proximidad física a pacientes (sintomáticos o asintomáticos) en un medio susceptible y propenso a los aerosoles. Nosotros ponemos las barreras físicas, podemos hacer pruebas de antígenos, incluso podríamos apoyar a la vacunación de todos. Somos personal de salud que podemos apoyar a la contención de la pandemia de COVID-19.

Es un hecho que al estar cerradas las universidades, las facultades y escuelas de odontología han dejado de prestar servicio a más de un millón de personas que asisten regularmente a recibir atención odontológica, desde una limpieza hasta tratamientos de rehabilitación. Lo mismo ha pasado con los derechohabientes de los servicios de salud pública, que no han recibido atención dental de urgencia o de seguimiento por los mismos recortes en horarios y personal.
\end{abstract}

Agustín Zerón*

\section{Acto de justicia}

Exigimos por derecho constitucional y sanitario no permanecer excluidos en la vacunación prioritaria ante el alto riesgo ocupacional, por la alta concentración a fuentes de contaminación biológica, y un alto riesgo de infección en la práctica médica pública y privada.

Lamentablemente la respuesta del presidente López Obrador: «los médicos particulares deben esperar para su vacuna, hasta que nos toque a todos» es impugnable, pues viola el derecho a la salud que está establecido en el art. $1^{\circ}$ párrafos tercero y quinto y art. $4^{\circ}$ párrafo cuarto de la Constitución Política de los Estados Unidos Mexicanos.

\section{SÍNTESIS DE CONSIDERACIÓN BIOMÉDICA}

\section{Organización Mundial de la Salud (OMS)}

Los trabajadores de salud tenemos una función fundamental no sólo en la atención clínica de los pacientes, sino también a la hora de velar para que se apliquen las debidas medidas de prevención y control de infecciones en los establecimientos sanitarios.

La Organización Mundial de la Salud ha realizado una evaluación de los factores de riesgo de enfermedad por el coronavirus de 2019 (COVID-19) entre trabajadores de salud donde:

* Editor en Jefe de la Revista ADM, Miembro de la Comisión de Honor y Justicia de la Asociación Dental Mexicana. México.

Citar como: Zerón A. Vacunación para odontólogos. Encuentros cercanos de primer contacto. Rev ADM. 2021; 78 (2): 62-72. https://dx.doi. org/10.35366/99280 
La definición de «trabajador de salud» no debe ser excesivamente restrictiva, de modo que quede incluido un gran número de miembros del personal potencialmente expuestos al contagio. Por esta razón, la definición de trabajador de salud debe englobar a todos los miembros del personal del establecimiento sanitario que hayan intervenido en la atención de algún paciente infectado por SARS-CoV-2 o con COVID-19.

En el personal de salud están incluidos no sólo los de la misma zona que el paciente, sino también aquellos que quizá no le hayan dispensado atención directa pero que han estado en contacto con líquidos corporales del paciente o bien con objetos o superficies ambientales potencialmente contaminados. Así pues, la definición debe incluir tanto a los profesionales sanitarios (médicos y odontólogos), los profesionales paramédicos y los trabajadores auxiliares, como al personal de limpieza y lavandería, radiólogos y técnicos de radiología, personal administrativo, flebotomistas, terapeutas respiratorios, nutricionistas, trabajadores sociales, fisioterapeutas, personal de laboratorio, limpiadores, personal de ingresos o recepción, transportistas de pacientes y personal de servicios de restauración, entre otros.

La exposición a pacientes con COVID-19 se define como sigue:

- El contacto estrecho (a menos de 1 metro de distancia y durante más de 15 minutos con uno o varios pacientes sospechosos/probables/confirmados de COVID-19);

- El contacto indirecto con fómites (por ejemplo, prendas de vestir, ropa de cama, utensilios, mobiliario, entre otros) o con materiales, dispositivos o equipo asociados con un paciente sospechoso/probable/ confirmado de COVID-19.

En los criterios de inclusión están todos los trabajadores de salud que hayan podido estar expuestos a la COVID-19 en un establecimiento en el que se esté prestando atención a un paciente con infección por el virus SARS-CoV-2 confirmada en el laboratorio, incluida la exposición a la sangre y los líquidos corporales del paciente y a materiales, dispositivos y equipo contaminados relacionados con el paciente.

\section{REFERENCIA}

1. Organización Mundial de la Salud. Evaluación de los factores de riesgo de enfermedad por el coronavirus de 2019 (COVID-19) entre trabajadores de salud: protocolo para un estudio de casos y testigos, versión 1.0. Organización Mundial de la Salud. 2020. Disponible en: https://apps.who.int/iris/handle/10665/332505.

\section{ENTENDIENDO LAS MUERTES DE TRABAJADORES DE SALUD MEXICANOS POR COVID-19}

La reconocida revista científica The Lancet y dos empresas británicas (Digital PR Kaizen y Lenstore) han presentado un estudio conjunto que determina cuáles son las profesiones más expuestas a riesgos de salud, entre los que se encuentran con más posibilidad de contagio de COVID-19. Centrándonos en el último apartado del reporte, se observó que los profesionales del área de la salud son, en líneas generales, los más expuestos a contagio por coronavirus, con ocho profesiones entre las 10 más arriesgadas en este aspecto. Las siete profesiones con un mayor riesgo de infección son, por este orden, odontólogos, enfermeros de cuidados intensivos, médicos de cabecera, paramédicos y técnicos de emergencia sanitaria, anestesiólogos, cirujanos y veterinarios, mientras que en el noveno sitio se sitúan los fisioterapeutas.

\section{REFERENCIA}

1. Agren D. Understanding Mexican health worker COVID-19 deaths. Lancet. 2020; 396 (10254): 807. doi: 10.1016/S01406736(20)31955-3.

\section{INMUNIZACIÓN A QUIENES NO ESTÁN EN LA «PRIMERA LÍNEA DE ATENCIÓN» PERO SÍ EN EL PRIMER CONTACTO DE ATENCIÓN A PACIENTES ASINTOMÁTICOS}

«El hecho de que más de 7,000 personas mueran en su intento por salvar vidas constituye una crisis a escala asombrosa».

Amnistía Internacional.

El gobierno debe garantizar que todo el personal sanitario como respuesta ante la COVID-19 tiene el mismo acceso a beneficios y protecciones, independientemente del carácter de su contrato. «La falta de protección adecuada a los trabajadores y trabajadoras de la salud no sólo socava sus derechos humanos y pone a estas personas en riesgo, sino que aumenta el peligro para todos los habitantes».

El Gobierno Federal es quien determina la Política Nacional de Vacunación y en esta administración apoyaría en la logística de aplicación. Sin embargo, la vacunación se ha negado a quienes no están en la «primera línea de 
atención», por lo que dependerá de las dosis «sobrantes» para se apliquen a los médicos particulares según lo indique la Secretaría de Salud.

La preocupación crece mientras aumenta el número de fallecimientos del personal de salud. Hoy, México es el país con el mayor número de muertes en las Américas, de acuerdo con la Organización Panamericana de la Salud (OPS) y en su Actualización Epidemiológica Enfermedad por Coronavirus (COVID-19) informa al menos 3,534 fallecimientos registrados desde enero de 2020 al 10 de marzo de 2021.

Si bien el año 2021 ha sido, merecidamente, designado como el Año Internacional de los Trabajadores Sanitarios y Asistenciales para reconocer y agradecer la inquebrantable dedicación de estos trabajadores a la lucha contra la pandemia de COVID-19, desafortunadamente, el número de casos y defunciones en este grupo continúa aumentando, habiéndose notificado 1,369,969 casos confirmados acumulados, incluidas 7,389 defunciones al 10 de marzo de 2021.

\section{FUENTE}

- PAHO OPS-OMS. Epidemiological Update: Coronavirus disease (COVID-19) (11 March 2021). Available in: https://iris.paho.org/ handle/10665.2/53381

\section{COVID-19: LAS MUERTES DE PERSONAL SANITARIO ASCIENDEN AL MENOS A 17,000, MIENTRAS LAS ORGANIZACIONES PIDEN UNA RÁPIDA DISTRIBUCIÓN DE LAS VACUNAS}

«Cada 30 minutos muere de COVID-19 algún trabajador de la salud. Es una tragedia y una injusticia. El personal sanitario de todo el mundo ha arriesgado su vida para intentar protegernos frente a la COVID-19 y, sin embargo, en demasiados casos lo han hecho sin contar con protección alguna, y han pagado el peor precio por ello», afirma Steve Cockburn, director de Justicia Económica y Social de Amnistía Internacional.

Al mismo tiempo, el personal sanitario de algunos países que ya han iniciado sus programas de vacunación corre el riesgo de no ser considerado un colectivo prioritario o de tener que esperar para vacunarse, debido bien a la falta de suministro, bien a problemas de ejecución de los planes de vacunación, o bien por una definición limitada del concepto de profesional de la salud.

En términos generales, el personal sanitario en Europa ha sido un grupo prioritario en el marco de los planes nacionales, pero los problemas de suministro han ralentizado el proceso de vacunación. En algunos países, los sindicatos también han tenido que abogar para que el personal de las residencias de mayores se incluyese en la definición de personal sanitario, de manera que también se contasen entre los colectivos prioritarios para la vacunación.

Amnistía Internacional reconoce el trabajo de multitud de sindicatos nacionales de personal sanitario que se han esforzado enormemente en elaborar listas detalladas al respecto para honrar la memoria de sus colegas, y agradece también la labor de organizaciones regionales como la Federación Sindical Europea de Servicios Públicos (FSESP) y sus afiliados, que han contribuido a la recogida de datos, de organizaciones mundiales como ISP y UNICARE, y de Medscape y otros proyectos de medios de comunicación que han dado seguimiento a los fallecimientos de personal sanitario, como Lost on the frontline de The Guardian y Kaiser Health News.

\section{México lidera muertes por COVID-19 en personal médico}

En México se contabilizaron 2,996 decesos entre su personal sanitario, el primer lugar dentro de una lista de 18 países. La cifra registrada en nuestro país duplica la del segundo lugar, ocupado por Estados Unidos con 1,347 defunciones, a pesar de que hay una diferencia significativa entre el número de contagios entre los profesionales de la salud: 393 mil en el país norteamericano por 219 mil en México (Tabla 1).

\begin{tabular}{|c|c|c|c|}
\hline País & Muertes & Contagios & $\begin{array}{c}\text { \% Tasa de } \\
\text { letalidad }\end{array}$ \\
\hline México & 2,996 & 219,180 & 1.3 \\
\hline Estados Unidos & 1,347 & 393,104 & 0.3 \\
\hline Perú & 589 & 30,675 & 1.9 \\
\hline Brasil & 480 & 457,686 & 0.1 \\
\hline Argentina & 446 & 75,317 & 0.6 \\
\hline Colombia & 201 & 39,241 & 0.5 \\
\hline Venezuela & 121 & 1,678 & 7.2 \\
\hline Ecuador & 114 & 11,038 & 1.0 \\
\hline Chile & 102 & 52,241 & 0.1 \\
\hline Guatemala & 84 & 9,141 & 0.9 \\
\hline
\end{tabular}




\section{FUENTE}

— Amnesty.Org, 5 marzo 2021, 00:01 UTC. Disponible en: https:// www.amnesty.org/es/latest/news/2021/03/covid19-health-workerdeath-toll-rises-to-at-least-17000-as-organizations-call-for-rapidvaccine-rollout/

\section{REFERENCIAS}

1. Amnistía Internacional. Global: el análisis de Amnistía revela que más de 7.000 trabajadores sanitarios han muerto a causa del COVID-19. Publicado el 3 de septiembre de 2020. Consultado en versión electrónica.

2. Organización Panamericana de la Salud. Rueda de prensa semanal sobre la situación de COVID-19 en la Región de las Américas. Consultado en versión electrónica.

3. Reina R, Chiappero G. Comunicado de la Sociedad Argentina de Terapia Intensiva a la Sociedad Argentina.

4. Universidad Nacional de La Plata. En el contexto dramático de una de las crisis sanitarias más graves de la historia argentina, profesores de la Facultad de Ciencias Médicas de la Universidad Nacional de La Plata quieren expresar su extrema preocupación por la situación actual. Consultado en versión electrónica.

5. Teixeira CFS, Soares CM, Souza EA, Lisboa ES, Pinto ICM, Andrade LR, Espiridião MA. The health of healthcare professionals coping with the Covid-19 pandemic. Cien Saude Colet. 2020; 25 (9): 3465-3474. doi: 10.1590/141381232020259.19562020.

6. Ayanian JZ. Mental health needs of health care workers providing frontline COVID-19 Care. JAMA Health Forum. 2020. doi: 10.1001/ jamahealthforum.2020.0397.

\section{CLASIFICACIÓN DE RIESGO ANTE LA EXPOSICIÓN AL SARS-CoV-2 Y COVID-19}

Esta resolución establece cuatro criterios de clasificación a la exposición al CoVID-19 según las actividades laborales: riesgo bajo o de precaución, riesgo mediano, riesgo alto y muy alto (Figura 1).

\section{Riesgo bajo}

Se entienden por actividades con riesgo bajo aquéllas que no requieren contacto con personas que se sabe o sospecha que están infectados de COVID-19, trabajadores con un contacto ocupacional mínimo con público y sus compañeros.

\section{Riesgo medio}

Por su parte, se consideran ocupaciones con riesgo medio de exposición los trabajadores que requieren un contacto frecuente o cercano (menos de 2 metros de distancia), con personas que podrían estar infectadas de COVID-19 pero que no son pacientes que se conoce o se sospecha estén contagiados.
Riesgo alto y muy alto

El riesgo alto y muy alto de exposición corresponden a ocupaciones con riesgo potencial de exposición o con contacto directo a fuentes conocidas o sospechosas de COVID-19. Por ejemplo: personal de la salud (médico y odontólogos).

\section{FUENTES}

- Riesgo de Exposición de los Trabajadores a COVID-19 Occupational Safety and Health Administration.

- Comprueba el riesgo de contagiarte de coronavirus según tu profesión - ABC España.

- O*NET Data.

- Departamento del Trabajo de los EE. UU. Administración de Seguridad y Salud Ocupacional OSHA 3992-03 2020. Disponible en: https://www.osha.gov/sites/default/files/publications/ OSHA3993SP.pdf

\section{OCUPACIÓN Y RIESGO DE COVID-19 SEVERO}

Los profesionales del área de la salud tienen siete veces más riesgo de contraer COVID que el resto de las profesiones. Hasta donde sabemos, este estudio es el más grande hasta la fecha para evaluar el riesgo de COVID-19 grave en todos los grupos ocupacionales. Encontramos un riesgo siete veces mayor para los trabajadores de la salud y un riesgo dos veces mayor para los trabajadores de la asistencia social y el transporte, en comparación con los trabajadores no esenciales. Aparte de los trabajadores del transporte, el ajuste por las covariables no alteró sustancialmente las asociaciones, lo que implica que las variables socioeconómicas, de salud, laborales y relacionadas con el estilo de vida estudiadas no fueron los principales factores mecanicistas que sustentan las diferencias ocupacionales.

El mayor riesgo encontrado entre los trabajadores del transporte parece deberse a factores socioeconómicos.

Las comparaciones del riesgo grave de COVID-19 entre los grupos ocupacionales de salud y asistencia social destacaron cómo estos riesgos más altos parecen estar particularmente vinculados a los trabajos, en lugar de reflejar circunstancias socioeconómicas más amplias.

Este estudio tiene varias fortalezas importantes. Primero, mediante el uso de un estudio de cohorte bien caracterizado, pudimos comparar el riesgo de infección en una amplia gama de grupos ocupacionales e identificar ocupaciones que pueden tener un mayor riesgo de COVID-19 grave. La vinculación de datos, el gran tamaño de la muestra y los datos 


\section{Riesgo de Exposición de los Trabajadores a COVID-19}

Dada la naturaleza evolutiva de la pandemia, OSHA está en proceso de revisar y actualizar este documento. Es posible que estos materiales ya no representen las recomendaciones y guias actuales de OSHA. Para obtener la información más actualizada, consulte la Guia de protección de los trabajadores.

Clasificando la Exposición de los Trabajadores al SARS-CoV-2

El riesgo de los trabajadores de exposición ocupacional al SARS-CoV-2, el virus que causa COVID-19, durante un brote depende en parte del tipo de industria y la necesidad de contacto a menos de 6 pies de las personas que se conoce o se sospecha que tienen COVID-19.

OSHA ha dividido las tareas de trabajo en cuatro niveles de exposición al riesgo, como se muestra a continuación. La mayoría de los trabajadores estadounidenses probablemente estarán en los niveles de riesgo de exposición más bajo (de precaución) o en los niveles de riesgo de exposición medio.

Pirámide de Riesgos Laborales para COVID-19 RIESGO MUY ALTO DE EXPOSICIÓN

Los trabajos con riesgo muy alto de exposición a fuentes conocidas o sospechosas de COVID-19 durante procedimientos médicos específicos, trabajos mortuorios o procedimientos de laboratorio Los trabajadores en esta categoría incluven:

- Trabajadores del cuidado de la salud y de morgues que realizan procedimientos generadores de aerosol o recopilando/ manipulando especímenes de pacientes potencialmente infecciosos o cuerpos de personas que se conoce o se sospecha que tienen COVID-19 al momento de muerte.

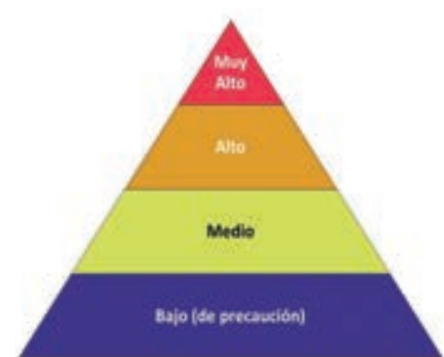

RIESGO ALTO DE EXPOSICIÓN

Los trabajos con un alto potencial de exposición a fuentes conocidas o sospechosas de COVID-19. Los trabajadores en esta categoría incluyen:

- Personal de apoyo y atención del cuidado de la salud, transportes médicos y trabajadores mortuorios expuestos a pacientes conocidos o sospechosos de COVID-19 o cuerpos de personas que se conoce o se sospecha que tienen COVID-19 en el momento de la muerte.

\section{RIESGO MEDIO DE EXPOSICIÓN}

Los trabajos que requieren contacto frecuente y/o cercano con personas que podrian estar infectadas, pero que no son pacientes conocidos o sospechosos. Los trabajadores en esta categoría incluyen

- Aquellos que pueden tener contacto con el público en general (por ej. escuelas, ambientes de trabajo de alta densidad poblacional, algunos ambientes de alto volumen comercial), incluyendo las personas que regresan de lugares con transmisión generalizada del COVID-19.

RIESGO BAJO DE EXPOSICIÓN (DE PRECAUCIÓN)

Los trabajos que no requieren contacto con personas que se conoce o se sospecha que están infectadas.

- Los trabajadores en esta categoría tienen un contacto ocupacional mínimo con el público y otros compañeros de trabajo

Para obtener más información, consulte la Guía sobre la Preparación de los Lugares de Trabajo para el virus COVID-19.

\section{OSHA $=$ • osha.gov/Covid-19 $\cdot 1$ 1-800-321-OSHA (6742) $\cdot$ @OSHA_DOL $y$}

detallados nos permitieron proporcionar rápidamente evidencia empírica de la pandemia en curso e investigar hasta qué punto los resultados observados se explican potencialmente por una amplia gama de factores (Figura 2).

\section{REFERENCIA}

1. Mutambudzi M, Niedwiedz C, Macdonald EB, Leyland A, Mair F, Anderson J et al. Occupation and risk of severe COVID-19: prospective cohort study of 120075 UK Biobank participants. Occup Environ Med. 2020. doi: 10.1136/oemed-2020-106731.

\section{Figura 1:}

Los odontólogos estamos en la zona de muy alto riesgo. Tomada de: https:// www.osha.gov/sites/default/files/ publications/OSHA3993SP.pdf

\section{ENTRE LA SEGURIDAD Y EL MIEDO SÍNDROME COVID Y POST-COVID}

Desde el inicio de la pandemia de COVID-19, el riesgo que plantean los procedimientos que generan aerosoles se ha convertido en un tema determinante en la odontología. Los profesionales de la salud oral nos enfrentamos a nuevos retos y un refuerzo de las medidas necesarias y recientes recomendaciones de bioseguridad, basadas en una comprensión en rápida evolución y variantes del SARS-CoV-2. 
A pesar de que el gremio odontológico ha estado siguiendo estrictas directrices de control de infecciones, desde mucho antes de COVID-19, el riesgo de contaminación está presente ante la virulencia del nuevo coronavirus. La Dirección Científica de la American Dental Association (ADA) presentó otro estudio como punto de prueba de que la atención dental es segura para los pacientes que acuden al consultorio dental.

Con la tasa de infección obtenida se alineó a la tasa de infección acumulada entre los odontólogos en comparación con otros grupos, incluyendo a las higienistas dentales. En la misma encuesta también se preguntó a los profesionales de la salud oral sobre su salud mental,

\section{A}

Risk of severe COVID-19 by detailed occupational groups of essential workers

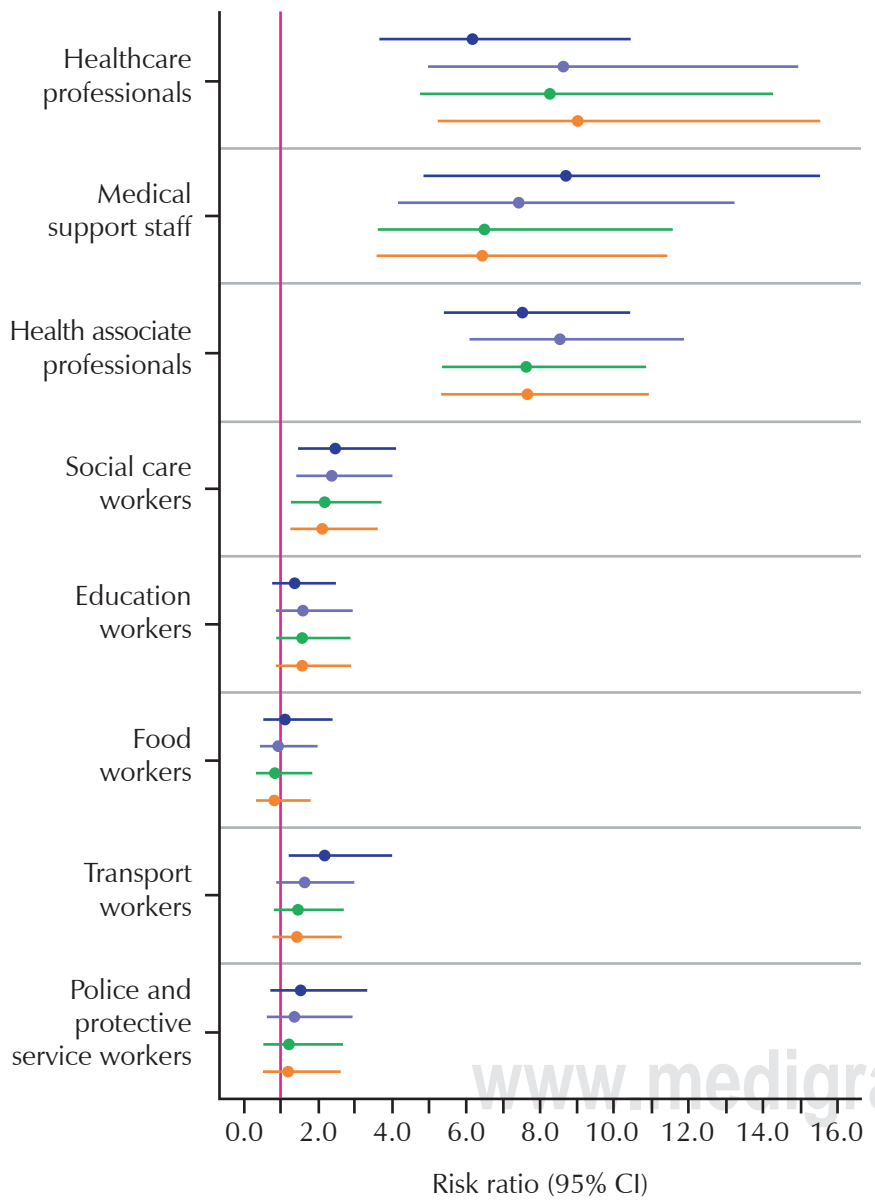

Reference: non-essential workers

- Sociodemographic variables

- Socioeconomic variables encontrando que el $25.7 \%$ había experimentado síntomas de ansiedad, y el $16.05 \%$ había experimentado síntomas de depresión. Los síntomas se asociaron con la edad de los participantes; los de 18 a 29 años informaron los niveles más altos de síntomas y los de 64 años o más informaron los más bajos niveles de ansiedad o depresión.

En otro estudio para comprender cómo los odontólogos en diferentes partes del mundo están lidiando con el miedo a contraer la COVID-19, y qué estrategias o modificaciones están implementando en sus clínicas para combatir el SARS-CoV-2, los investigadores han destacado los devastadores efectos psicológicos que la COVID-19

B

Risk of severe COVID-19 by SOC2000 major occupational groups

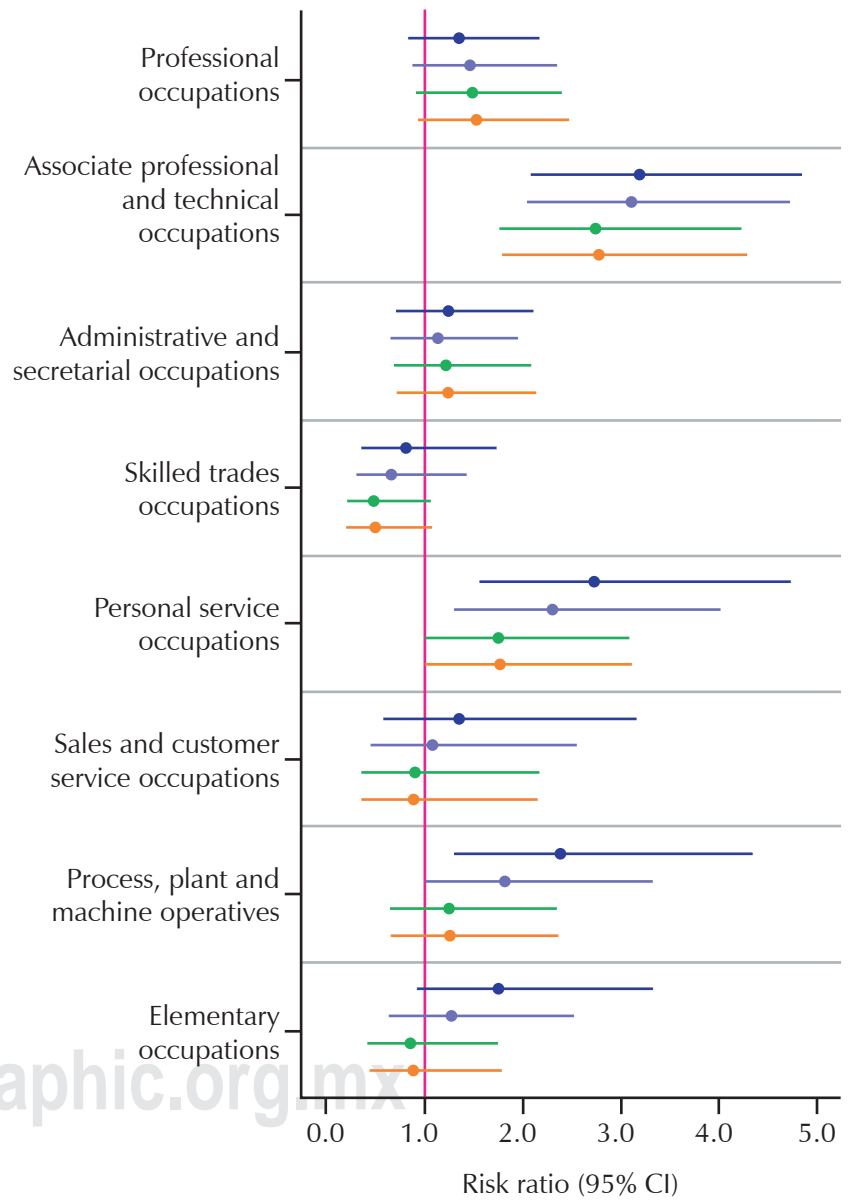

Reference: Managers and senior officials

- + Work-related variables $\quad$ All variables

Figura 2: Tabla de riesgo ocupacional. Tomada de: Mutambudzi M et al. 
ha tenido en los profesionales de la salud oral. De los 650 odontólogos encuestados de 30 países, la mayoría (90\%) conocía los cambios recientes en los protocolos de tratamiento, pero sólo el $61 \%$ dijo que sus prácticas sí han modificado los protocolos de tratamiento para el control de infecciones como resultado de la pandemia.

Es interesante notar que, a pesar de tener un alto nivel de conocimiento, los odontólogos de todo el mundo viven en un estado de miedo constante de contraer la enfermedad mientras realizan tratamientos dentales de rutina, VIVIMOS EN PSICOENDEMIA. Esta ansiedad ha llevado a la modificación de sus prácticas u horas de servicio y a la provisión de atención dental de emergencia únicamente. Algunos de los odontólogos han tenido que cerrar sus consultorios por tiempo indefinido hasta que la situación esté bajo control de forma segura.

\section{REFERENCIA}

1. Ahmed MA, Jouhar R, Ahmed N, Adnan S, Aftab M, Zafar MS et al. Fear and practice modifications among dentists to combat novel coronavirus disease (COVID-19) outbreak. Int J Environ Res Public Health. 2020; 17 (8): 2821. doi: 10.3390/ijerph17082821.

\section{AEROSOLES Y SARS-CoV-2}

Existe evidencia abrumadora de que la inhalación del coronavirus y el síndrome respiratorio agudo severo (SARS-CoV-2) representan la ruta de transmisión más importante para la enfermedad COVID-19. Existe una necesidad urgente de armonizar los debates sobre los modos de transmisión del virus entre las disciplinas para garantizar mejores y más efectivas estrategias de control, y proporcionar una orientación clara y coherente al público. Para ello, debemos aclarar la terminología para distinguir entre aerosoles y gotitas utilizando un umbral de tamaño de $100 \mu \mathrm{m}$, no los $5 \mu \mathrm{m}$ históricos. Este tamaño separa de manera más efectiva su comportamiento aerodinámico, la capacidad de ser inhalado y la eficacia de las intervenciones para detener la propagación viral.

Las partículas de $10 \mu \mathrm{m}$ o menos (PM2.5) pueden ser inhaladas o permanecer en bioaerosoles durante horas en espacios de poca ventilación. Las bacterias tienen un tamaño de 1 a $3 \mu \mathrm{m}$ y un coronavirus de 0.1 a $0.5 \mu \mathrm{m}$. Los odontólogos trabajamos en la boca del paciente, expuestos a sangre, saliva y bioaerosoles.

Los virus se transmiten en gotitas (mayores de $100 \mu \mathrm{m}$ ) que caen en segundos comúnmente al suelo a 2 metros de la fuente expulsora y pueden rociarse como pequeñas balas de cañón sobre individuos cercanos. Debido a su rango de recorrido limitado, el distanciamiento físico reduce la exposición a estas gotas. También existen virus en bioaerosoles (menores de
$100 \mu \mathrm{m})$, pueden permanecer suspendidos en el aire durante muchos minutos, hasta horas, como el humo, que pueden ser inhalados. Los coronavirus están muy concentrados cerca de una persona infectada, por lo que pueden infectar más fácilmente a las personas cercanas. Pero los bioaerosoles que contienen virus infecciosos también pueden viajar más de 2 metros y acumularse en el aire interior mal ventilado, lo que lleva a eventos de superpropagación.

\section{REFERENCIAS}

1. Holliday R, Allison JR, Currie CC, Edwards DC, Bowes C, Pickering K et al. Evaluating contaminated dental aerosol and splatter in an open plan clinic environment: Implications for the COVID-19 pandemic. J Dent. 2021; 105: 103565. doi: 10.1016/j.jdent.2020.103565.

2. Prather KA, Marr LC, Schooley RT, McDiarmid MA, Wilson ME, Milton DK. Airborne transmission of SARS-CoV-2. Science. 2020; 370 (6514): 303-304.

\section{UN ESTUDIO SIMULA CÓMO SE COMPORTA EL CORONAVIRUS SARS- COV-2 EN AEROSOLES Y SUPERFICIES ESTUDIOS AEROSOLES COCHRANE COVID-19}

\section{9 marzo 2020 | Centro Cochrane Iberoamericano}

Los virus en aerosoles (menores de $100 \mu \mathrm{m}$ ) pueden permanecer suspendidos en el aire durante muchos segundos u horas, como el humo, y ser inhalados. Están altamente concentrados cerca de una persona infectada, por lo que pueden infectar más fácilmente a las personas que están cerca. Pero los aerosoles que contienen virus infecciosos también pueden viajar más de $2 \mathrm{~m}$ y acumularse en el aire interior mal ventilado, lo que conduce a eventos de supercontagio. Los individuos con COVID-19, muchos de los cuales no tienen síntomas, liberan miles de aerosoles cargados de virus y muchas menos gotitas al respirar y hablar. Por lo tanto, es mucho más probable que uno inhale aerosoles a que sea alcanzado por una gota. Y, por lo tanto, el equilibrio de la atención debe cambiarse a la protección contra la transmisión aérea.

\section{REFERENCIA}

1. van Doremalen N, Bushmaker T, Morris DH, Holbrook MG, Gamble A, Williamson BN et al. Aerosol and surface stability of SARS-CoV-2 as compared with SARS-CoV-1. N Engl J Med. 2020; 382 (16): 1564-1567. doi: 10.1056/NEJMc2004973.

\section{REPORTE DE LA OMS}

Vías de transmisión del virus de la COVID-19: repercusiones para las recomendaciones relativas a las precauciones en materia de prevención y control de las infecciones. 
La transmisión por gotículas (Flügge) es distinta de la transmisión aérea, pues esta última tiene lugar a través de núcleos goticulares que contienen diversos microbios. Los núcleos goticulares, que tienen un diámetro inferior a $5 \mu \mathrm{m}$, pueden permanecer en el aire durante periodos prolongados y llegar a personas que se encuentren a más de un metro de distancia.

\section{FUENTE}

- OMS. Reseña científica. 29 de marzo de 2020. Disponible: https://www.who.int/es/news-room/commentaries/detail/modesof-transmission-of-virus-causing-covid-19-implications-for-ipcprecaution-recommendations

\section{REFERENCIAS}

1. Organización Mundial de la Salud. Prevención y control de las infecciones respiratorias agudas con tendencia epidémica y pandémica durante la atención sanitaria. Ginebra: Organización Mundial de la Salud; 2014. Disponible en: https://www.paho.org/hq/dmdocuments/2014/2014cha-prevencion-control-atencion-sanitaria.pdf

2. Liu J, Liao X, Qian S, Yuan J, Wang F, Liu Y et al. Community transmission of severe acute respiratory syndrome coronavirus 2 , Shenzhen, China, 2020. Emerg Infect Dis. 2020; 26 (6): 1320-1323. doi: 10.3201/eid2606.200239.

3. Chan JF, Yuan S, Kok KH, To KK, Chu H, Yang J et al. A familial cluster of pneumonia associated with the 2019 novel coronavirus indicating person-to-person transmission: a study of a family cluster. Lancet. 2020; 395 (10223): 514-523. doi: 10.1016/S0140-6736(20)30154-9.

4. Li Q, Guan X, Wu P, Wang X, Zhou L, Tong Y et al. Early transmission dynamics in Wuhan, China, of novel coronavirus-infected pneumonia. N Engl J Med. 2020; 382 (13): 1199-1207. doi: 10.1056/NEJMoa2001316.

5. Huang C, Wang Y, Li X, Ren L, Zhao J, Hu Y et al. Clinical features of patients infected with 2019 novel coronavirus in Wuhan, China. Lancet. 2020; 395 (10223): 497-506.

6. Burke RM, Midgley CM, Dratch A, Fenstersheib M, Haupt T, Holshue $\mathrm{M}$ et al. Active monitoring of persons exposed to patients with confirmed COVID-19 - United States, January-February 2020. MMWR Morb Mortal Wkly Rep. 2020; 69 (9): 245-246. doi: 10.15585/mmwr.mm6909e1.

7. Organización Mundial de la Salud. Informe de la misión conjunta OMS-China sobre la enfermedad por coronavirus 2019 (COVID-19) 16-24 de febrero de 2020 [Internet]. Ginebra: Organización Mundial de la Salud; 2020. Disponible en: https://www.who.int/ docs/default-source/coronaviruse/who-china-joint-mission-oncovid-19-final-report.pdf

8. Ong SWX, Tan YK, Chia PY, Lee TH, Ng OT, Wong MSY et al. Air, surface environmental, and personal protective equipment contamination by severe acute respiratory syndrome coronavirus 2 (SARS-CoV-2) from a symptomatic patient. JAMA. 2020; 323 (16): 1610-1612. doi: 10.1001/jama.2020.3227.

9. Zhang Y, Chen C, Zhu S, Shu C. Isolation of 2019-nCoV from a stool specimen of a laboratory-confirmed case of the coronavirus disease 2019 (COVID-19). China CDC Weekly. 2020; 2 (8): 123-124.

10. van Doremalen N, Bushmaker T, Morris DH, Holbrook MG, Gamble A, Williamson BN et al. Aerosol and surface stability of sars-cov-2 as compared with SARS-CoV-1. N Engl J Med. 2020; 382 (16): 1564-1567. doi: 10.1056/NEJMc2004973.
11. Cheng VCC, Wong SC, Chen JHK, Yip CCY, Chuang VWM, Tsang OTY et al. Escalating infection control response to the rapidly evolving epidemiology of the coronavirus disease 2019 (COVID-19) due to SARS-CoV-2 in Hong Kong. Infect Control Hosp Epidemiol. 2020; 41 (5): 493-498.

12. Orientaciones técnicas de la OMS para el control y la prevención de las infecciones en el contexto de la COVID-19. Disponible en: https:// www.who.int/emergencies/diseases/novel-coronavirus-2019/ technical-guidance/infection-prevention-and-control

13. Alhazzani W, Moller MH, Arabi YM, Loeb M, Gong MN, Fan E et al. Surviving sepsis campaign: guidelines on the management of critically ill adults with Coronavirus Disease 2019 (COVID-19). Intensive Care Med. 2020; 46 (5): 854-887. doi: 10.1007/s00134020-06022-5.

14. Interim guidelines for the clinical management of COVID-19 in adults. Australasian Society for Infectious Diseases Limited (ASID). Available in: https://www.asid.net.au/documents/item/1873

15. Coronavirus disease (COVID-19): for health professionals. Available in: https://www.canada.ca/en/public-health/services/diseases/2019novel-coronavirus-infection/health-professionals.html

16. Guidance on infection prevention and control for COVID-19. Available in: https://www.gov.uk/government/publications/wuhannovel-coronavirus-infection-prevention-and-control

17. Interim Infection Prevention and Control Recommendations for Patients with Suspected or Confirmed Coronavirus Disease 2019 (COVID-19) in Healthcare Settings. Available in: https://www.cdc.gov/coronavirus/2019ncov/infection-control/control-recommendations.html

18. Infection prevention and control for COVID-19 in healthcare settings. Available in: https://www.ecdc.europa.eu/en/publicationsdata/infection-prevention-and-control-covid-19-healthcare-settings

19. Rational use of PPE for COVID-19. Available in: https://apps. who.int/iris/bitstream/handle/10665/331498/WHO-2019-nCoVIPCPPE_use-2020.2-eng.pdf

20. Ran L, Chen X, Wang Y, Wu W, Zhang L, Tan X. Risk factors of healthcare workers with coronavirus disease 2019: a retrospective cohort study in a designated hospital of Wuhan in China. Clin Infect Dis. 2020; 71 (16): 2218-2221. doi: 10.1093/cid/ciaa287.

21. Infection Prevention and Control (IPC) for Novel Coronavirus (COVID-19) Course. Available in: https://openwho.org/courses/ COVID-19-IPC-EN

\section{PAPEL DEL ODONTÓLOGO ANTE LA COVID-19}

En la actualidad, existen suficientes referencias científicas para involucrar a la salud oral con la salud sistémica. Las enfermedades infecciosas en la cavidad oral tienen una relación bidireccional con diversos sistemas orgánicos. Una gran cantidad de investigación ha respaldado una conexión entre las enfermedades periodontales (periodontitis) y otras enfermedades sistémicas como la diabetes, enfermedades cardiovasculares, pulmonares, artritis reumatoide, Alzheimer, etcétera. Además, la evidencia científica emergente sugiere que una periodontitis podría predisponer a los pacientes a tener síntomas más graves de COVID-19. Por lo tanto, no sólo atendemos las importantes urgencias dentales, también ayudamos a mantener 


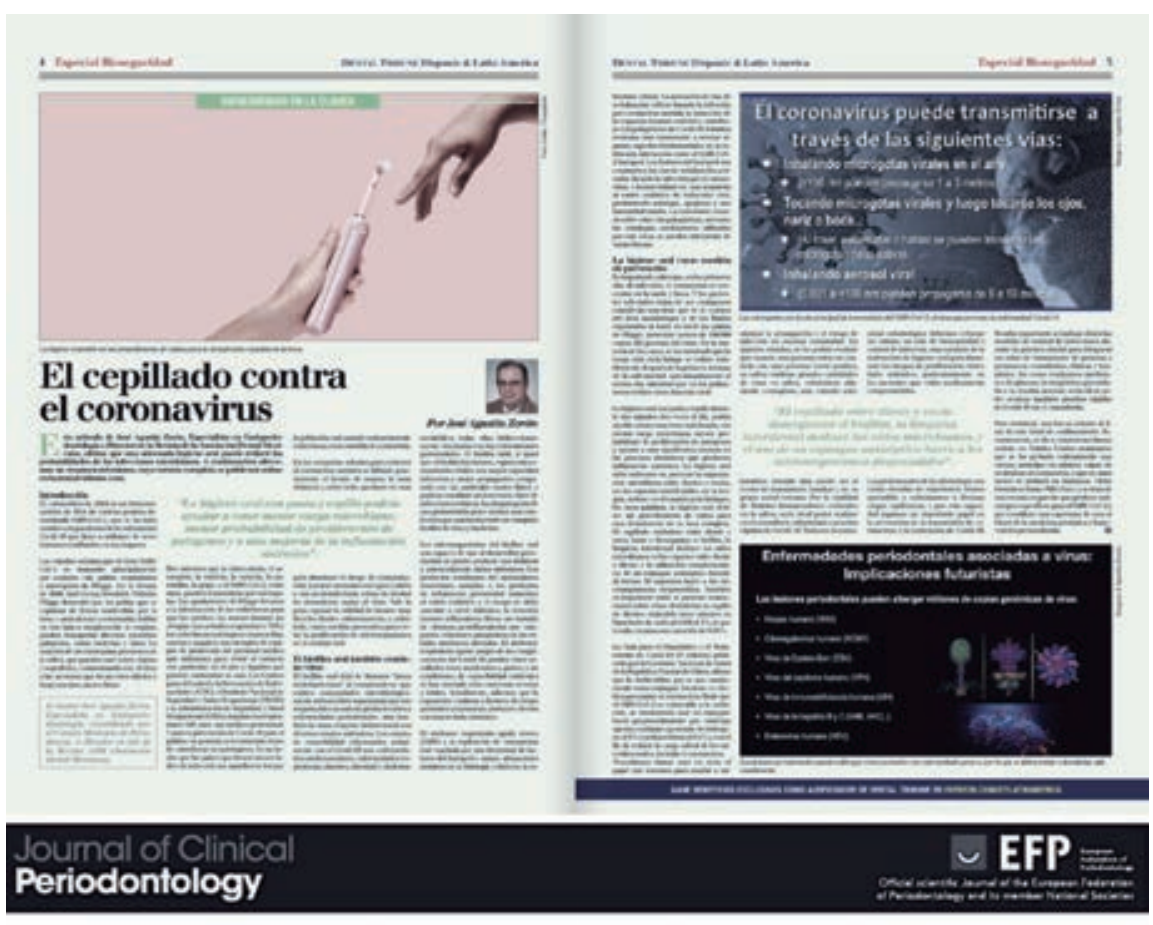

JRIGINAL ARTICLE CLINICAL PERIODONTOLOGY |

\section{Association between periodontitis and severity of COVID-19 infection: a case-control study}

Vadya Marouf, Wenji Cal, Khalid N. Said, Hanin Daas, Hanan Diab, Venkateswara Rao Chinta, Ali Ait Issain, Belinda Nicolau, Mariano Sanz, Faleh Tamimies

First published: 01 February 2021 | https://doi.org/10.1111/jepe.13435

This article has been accepted for publication and undergone full peer review but has not been through the copyediting, typesetting, pagination and proofreading process, which may lead to differences retween this version and the Version of Record. Please cite this article as doi:10.1111/jcpe.13435

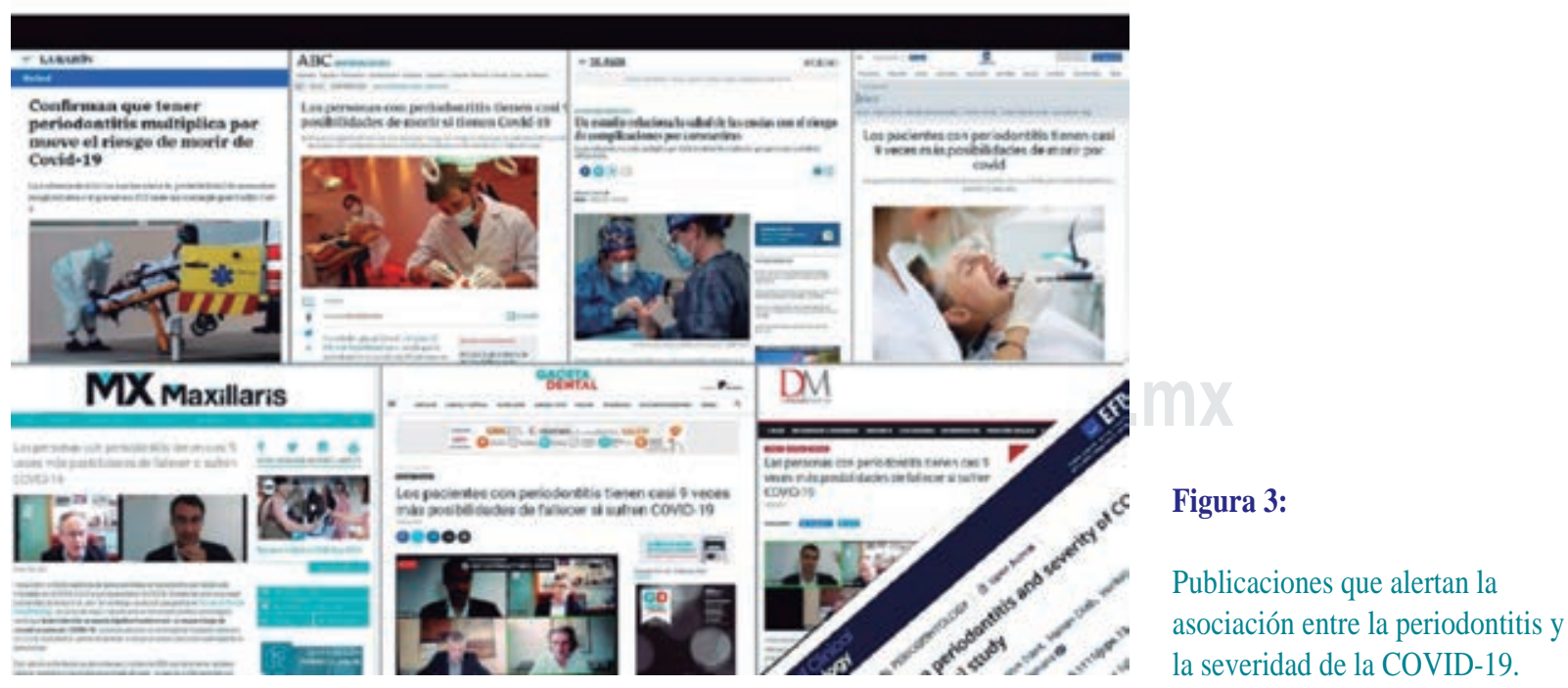




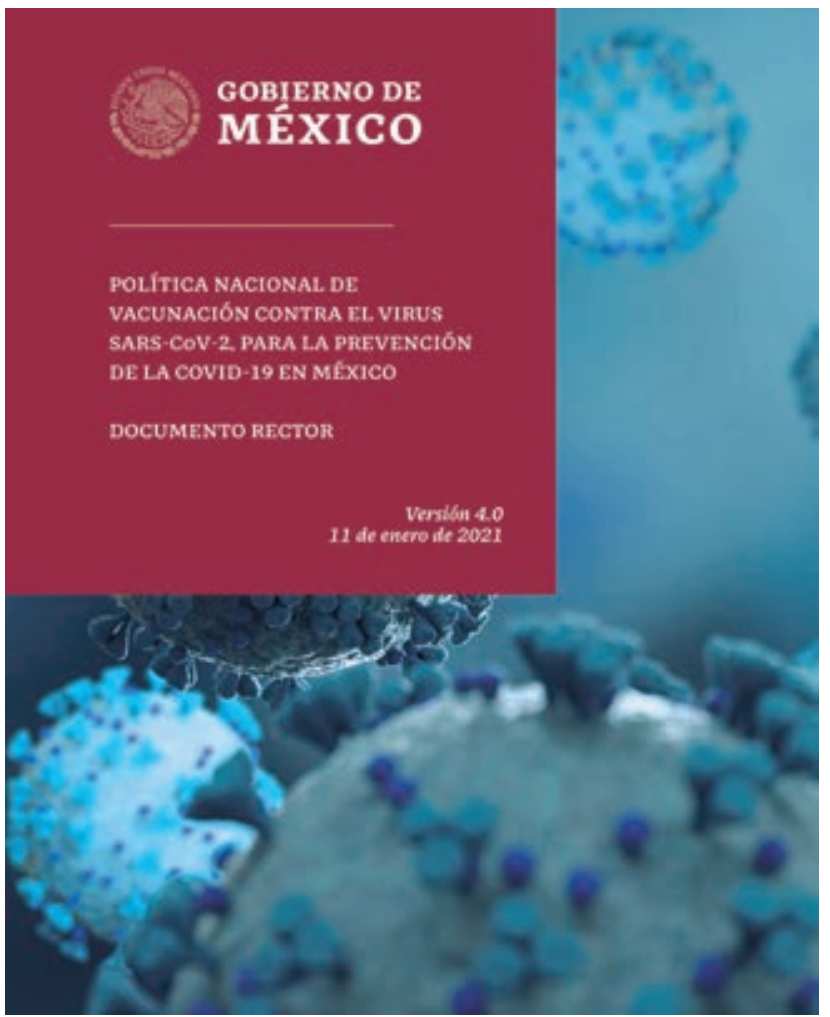

Figura 4: Portada del documento rector de la Política nacional de vacunación contra el virus SARS-CoV-2. Tomada de: https:/coronavirus.gob.mx/wp-content/uploads/2021/01/PolVx_COVID_11Ene2021.pdf

la salud oral, particularmente la salud periodontal, que hoy es más importante que nunca.

Estos hallazgos indican claramente lo serio y apasionado que es ejercer la profesión odontológica, que ha aceptado de manera responsable la importancia de garantizar la salud de sus pacientes y personal frente a la pandemia de COVID-19. Esto refuerza nuestro enfoque ante los procedimientos quirúrgicos y no quirúrgicos donde siempre se ha enfocado a garantizar que existan barreras de bioseguridad efectivas para nuestros pacientes, y ante la llegada del coronavirus hemos mejorado significativamente nuestras medidas de seguridad para tratar de prevenir la aparición y dispersión de la COVID-19.

Es de vital importancia que los pacientes se den cuenta de que es seguro visitar a los profesionales de la odontología, en especial a los periodontólogos. Por lo tanto, mantener la salud periodontal es más importante que nunca. Muchas de nuestras prácticas están enfocadas a disminuir la carga microbiana patógena para disminuir el riesgo de complicaciones sistémicas. Por lo que el cuidado a nuestros pacientes nunca había sido mayor, lo que significa que todos deben estar recibiendo un mensaje sobre la importancia de mantener dientes y encías sanos durante esta pandemia mundial.

En febrero de 2021 se publicó un importante estudio multicentro (Instituto Hamad de Qatar, Universidad McGill de Quebec y Universidad Complutense de Madrid) donde subrayan que la COVID-19 se asocia con una respuesta inflamatoria exacerbada que puede resultar en desenlaces fatales. Esta inflamación sistémica (tormenta de citocinas) también es una característica principal de la periodontitis. Se asoció que los pacientes con periodontitis tienen riesgo mayor de ingresar a una Unidad de Cuidados Intensivos, a la necesidad de ventilación asistida, incluso tener nueve veces más probabilidades de morir por una COVID-19 severa, debido al aumento de los niveles sanguíneos de biomarcadores ahora relacionados con peor pronóstico de la enfermedad por SARS-CoV-2. Los coronavirus pueden entrar a través de múltiples receptores (AC2) presentes en tejidos y glándulas salivales de la cavidad oral. Los principales hallazgos de estudios recientes revelan que la periodontitis podría ser

TRABAJO

SALUD

\section{CLASIFICACIÓN DEL RIESGO DE LOS TRABAJADORES POR OCUPACIÓN O CONDICIÓN DE VULNERABILIDAD}

\begin{abstract}
Para efectos del COVID-19, esta Cuîa identifica cuatro niveles de riespo en razón de la cercanla del trabajador con personas infectadas, o del nivel de contacto repetido o extendido con fuentes posibles de contaglo con motivo de su trabaja. Adernss, proporciona elementos para identificar a la población trabajadora
\end{abstract} vulnerable poe condiciones ajenas al riesgo ocupacional.

Tabla 2. Clasificación de riesgos por exposición en los Centros de Trabajo

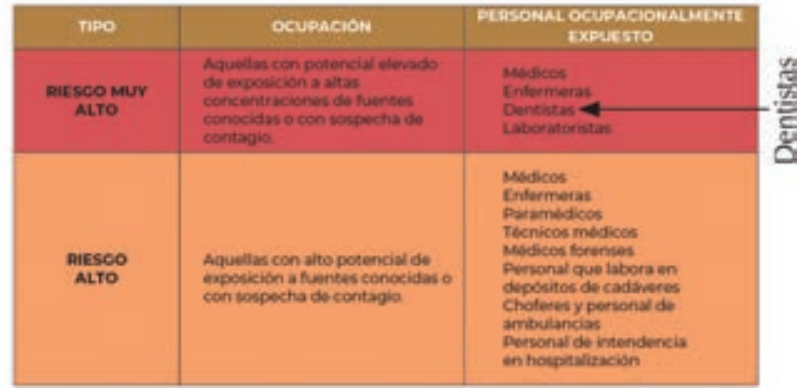

Figura 5: Clasificación del riesgo muy alto del personal odontológico. Tomada de: https://www.gob.mx/cms/uploads/attachment/ file/548062/GUI_A_DE_ACCIO_N_PARA_LOS_CENTROS_DE_ TRABAJO_ANTE_EL_COVID-19_24_04_20_VF.pdf 
un factor de riesgo de complicaciones por COVID-19. La higiene oral adecuada, las visitas frecuentes al odontólogo y un diagnóstico temprano con un tratamiento oportuno ayudarán a mejorar las condiciones generales de salud y a mitigar más rápidamente a esta pandemia (Figura 3).

Por la preparación profesional, y el equipamiento de un consultorio dental privado, los odontólogos podríamos estar realizando pruebas de antígenos a todos los pacientes para un diagnóstico temprano de infección por SARS-CoV-2 con impacto directo en la prevención y mitigación de la pandemia de COVID-19.

\section{REFERENCIA}

1. Marouf N, Cai W, Said KN, Daas H, Diab H, Chinta VR et al. Association between periodontitis and severity of COVID-19 infection: A case-control study. J Clin Periodontol. 2021. doi: 10.1111/jcpe.13435.

\section{CONSIDERACIONES LEGALES}

De acuerdo con la Política Nacional de vacunación contra el SARS-CoV-2 con fecha de publicación el 11 de enero del 2021. https://coronavirus.gob.mx/wp-content/ uploads/2021/01/PolVx_COVID_-11Ene2021.pdf

Donde su objetivo general marca:

1. Disminuir la carga de enfermedad y defunciones ocasionadas por la COVID-19.

Y objetivo específico:

1. Inmunizar al $100 \%$ del personal de salud que trabaja en la atención de la COVID-19.

Constitucionalmente (Art. 4) toda persona tiene derecho a la protección de la salud. Y faltando a este principio, existe una reiterada omisión para la instrumentación de una política de vacunación universal, integral e incluyente a favor del personal de la salud del sector privado.

Bajo esta omisión, consideramos que existe discriminación y notable violación al derecho humano de igualdad de manera directa (Política Nacional de Vacunación) e indirecta (atención que se brinda a pacientes sintomáticos o asintomáticos).*

1. La igualdad formal o de derecho:

a. Protección contra distinciones o tratos arbitrarios compuestos a su vez por la igualdad ante la ley como uniformidad en la aplicación de la norma jurídica por parte de todas las autoridades.
2. La igualdad sustantiva o de hecho:

a. Alcanzar una paridad de oportunidades en el goce del ejercicio real y efectivo de los derechos humanos.

* 1a. Sala SCJN- Jurisprudencia https://www.scjn.gob.mx/

Los odontólogos en general y particularmente los que estamos colegiados y certificados trabajamos en riguroso apego a la normatividad vigente (NOM-013-SSA2-2015) y Ley General de Salud (Art. 5), brindando atención estomatológica bajo los principios de bioseguridad (OMSOSHA) y precaución estándar (NOM) al considerar a todo paciente potencialmente infeccioso. Somos quienes recibimos a pacientes en primer contacto, y sin excepción alguna, atendemos cotidianamente a todos sin discriminación, estando expuestos continuamente a pacientes asintomáticos infectados de SARS-CoV-2 (Figuras 4 y 5).

\section{CONCLUSIÓN}

Es nuestro interés legítimo promover un juicio de amparo indirecto y colectivo con carácter de urgente por las violaciones a los derechos humanos:

1. Violación directa e indirecta al derecho de igualdad.

2. La omisión reiterada en la instrumentación de la Política Nacional de Vacunación para los profesionales sanitarios del sector privado.

3. Violación al derecho humano a la protección de la salud del personal de la salud del sector privado que también es vulnerable al contagio de COVID-19.

4. La vacunación prioritaria debe ser a grupos de riesgo que trabajan en la primera línea COVID, y NO se debe excluir a los profesionales de la salud pública y privada que tenemos encuentros cercanos de PRIMER CONTACTO con pacientes asintomáticos de SARS-CoV-2.

5. La OMS lo ha aclarado en su enfática respuesta: la vacuna debe ser para TODO el personal de salud. TODO EL PERSONAL DE SALUD, SON LOS DEL SECTOR PRIVADO Y PÚBLICO.

\section{FUENTES DE CONSULTA}

- Marouf N, Cai W, Said KN, Daas H, Diab H, Chinta VR et al. Association between periodontitis and severity of COVID-19 infection: A casecontrol study. J Clin Periodontol. 2021. doi: 10.1111/jcpe.13435.

— Defensa Inteligente. Disponible en: https://defensainteligente.com

- https://twitter.com/gsotomayorgva status $/ 1381727201767350275 ? \mathrm{~s}=20$

Correspondencia:

Dr. Agustín Zerón

E-mail: periodontología@hotmail.com 